\title{
Linewidth of a Semiconductor Laser Operating Near Threshold
}

\author{
Rongqing Hui, Nunzio Caponio, Sergio Benedetto, and Ivo Montrosset
}

\begin{abstract}
The linewidth of a single-mode semiconductor laser operating in the threshold region has been studied both theoretically and experimentally. Due to strong phase-amplitude coupling in a semiconductor laser, its linewidth versus current characteristics exhibits a local minimum below threshold and a local maximum just above threshold. This implies a limitation in the minimum optical bandwidth achievable in a resonant-type semiconductor laser optical amplifier. The theoretical prediction has been verified experimentally on a conventional DFB semiconductor laser.
\end{abstract}

\section{INTRODUCTION}

$\mathrm{S}$ EMICONDUCTOR lasers have been a subject of great interest for more than a decade now. If a semiconductor laser operates below threshold as a resonant optical amplifier, the noise bandwidth is determined by the Schawlow-Townes formula [1]. On the other hand, if a semiconductor laser operates above threshold, the spectral linewidth follows the modified Schawlow-Townes formula, in which the linewidth enhancement factor plays an important role [2], [3]. The linewidth characteristic of gas lasers has been calculated in the transition region from below to above threshold [4]-[6], but to our knowledge this has not been done yet for semiconductor lasers.

Recently, much attention has been paid to the resonant semiconductor laser amplifier used as a narrowband optical filter [7]-[10]. In this application, a laser is usually biased very near the threshold to achieve a narrow optical bandwidth. Therefore, it is necessary to have a better understanding of the laser linewidth behavior near threshold, since a laser filter's small-signal optical bandwidth is proportional to its noise bandwidth [10], [11]. In this letter, we present a theoretical description of a single mode semiconductor laser operating in the threshold region using the Fokker-Planck equation method [12]. The theoretical result has then been verified experimentally on a conventional distributed feedback (DFB) semiconductor laser. Because of the strong phase-amplitude coupling, a semiconductor laser has a different linewidth perfor-

Manuscript received March 6, 1992; revised May 7, 1992. This work was supported in part by CNR, Camera di Commercio di Torino under Research Grant "All-Optical Communication Networks" and the "Progetto Finalizzato Telecommunicazioni."

R. Hui, S. Benedetto, and I. Montrosset are with the Dipartimento di Elettronica, Politecnico di Torino, C. Duca degli Abruzzi 24, 10129 Torino, Italy.

N. Caponio is with the CSELT-Centro Studi e Laboratori Telecommunicazioni S.p.A., Via G. Reiss Romoli 274, 10148 Torino, Italy.

IEEE Log Number 9201816. mance as compared with a gas laser where this coupling is negligible. It is usually believed that the optical bandwidth of a semiconductor laser amplifier is monotonically decreasing as its bias current approaches threshold [7]-[9]. From our analysis we will show, however, that this smallsignal bandwidth decrease with the injection current is not monotonic. The minimum optical bandwidth has been achieved at the bias current below the threshold point. The value of the minimum optical bandwidth is determined by the spontaneous emission rate as well as the linewidth enhancement factor.

\section{THEORETICAL MODEL}

It is commonly accepted that the performance of a semiconductor laser can be well described by a set of Langevin rate equations for the complex field $E$ and the carrier population $N$, respectively [2]:

$$
\begin{gathered}
d E(t) / d t=\frac{\Delta G(N)}{2}(1+i \alpha) E(t)+F_{E}(t) \\
d N(t) / d t=I / q v-N / \tau_{e}-G(N) P+F_{N}(t)
\end{gathered}
$$

where $G(N)=G_{N}\left(N-N_{0}\right)$ is the modal gain, $G_{N}$ is the differential gain, $N_{0}$ is the carrier population at transparency, $\Delta G(N)=G(N)-\alpha_{0}$ is the gain margin, $\alpha_{0}$ is the loss coefficient, $\alpha$ is the linewidth enhancement factor, $I$ is the injection current, $P \propto|E|^{2}$ is the photon number, $v$ is the cavity volume, $\tau_{\varepsilon}$ is the effective carrier life time, and $q$ is the electron charge. $F_{E}(t)$ and $F_{N}(t)$ are the Langevin forces for the field and the carrier number, the latter can usually be omitted because the shot noise contribution to the linewidth is negligible [2]. The Langevin force for the field satisfies the correlation relation:

$$
\left\langle F_{E}(t) F_{E} *(u)\right\rangle=R \delta(t-u)
$$

where $\delta$ represents a delta function, $R=B \cdot B_{\mathrm{sp}} N^{2} / v$ is the spontaneous emission rate, $B$ is the radiative recombination rate and $B_{\mathrm{sp}}$ is the spontaneous emission factor indicating the fraction of the spontaneously emitted photons that coupled into the laser mode. In the mean-field approximation used in (1) and (2), the nonlinear gain and the spatial-hole-burning effects have been omitted. This is valid because in the low power condition, these contributions are negligible. Since in this letter we consider the linewidth, which is related only to the low frequency part of the field fluctuation, we can use the adiabatic approximation of the carrier population. This implies to assume 
that the population variation adiabatically follows the instantaneous variation in the photon number. With this approximation, (2) reduces to a simple form:

$$
G \approx G_{0}\left(1-P / P_{s}\right)
$$

where $G_{0}=G_{N}\left[I \tau_{e} /(q v)-N_{0}\right]$ is the small signal gain, $P_{s}=\left(G_{N} \tau_{e}\right)^{-1}$ is the saturation photon number and in (4) $P \ll P_{s}$ is assumed because in this analysis we are interested only in the laser behavior in the threshold region. Substituting (4) into (1) the correlation function $\langle E(t-$ $\left.\tau) E^{*}(t)\right\rangle$ of the complex field can be calculated by the Fokker-Planck equation technique [4]-[6]:

$$
\left\langle E(t-\tau) E^{*}(t)\right\rangle=\langle P\rangle \exp \left[-a \Lambda_{0} \tau\right]
$$

where $a=\left[R G_{0} /\left(8 P_{s}\right)\right]^{1 / 2}$ and $\Lambda_{0}$ is the complex eigenvalue with the lowest real part satisfying the nonhermitian eigenvalue equation [3], [4]:

$$
\begin{aligned}
\left\{\frac{d^{2}}{d r^{2}}-\frac{1}{4} r^{6}\right. & +\frac{1}{2} x r^{4}+\left(2-\frac{1}{4} x^{2}\right) r^{2} \\
& \left.-x+\frac{3}{4 r^{2}}-i \alpha\left(r^{2}-x\right)\right\} U_{n}=-\Lambda_{n} U_{n}
\end{aligned}
$$

where $x=\left(G_{0}-\alpha_{0}\right)\left[2 P_{s} /\left(R G_{0}\right)\right]^{1 / 2}$ is dependent on the injection current. The linewidth is determined by

$$
\Delta \nu=a \operatorname{Re}\left(\Lambda_{0}\right) / \pi
$$

where $\operatorname{Re}\left(\Lambda_{0}\right)$ represents the real part of $\Lambda_{0}$.

Equation (6) is solved numerically and the linewidth versus the injection current can be evaluated, the results are shown in Fig. 1. The linewidths predicted by the Schawlow-Townes formula $(\Delta \nu=R /(2 \pi P))$ for below threshold operation and by the modified SchawlowTownes formula $\left(\Delta \nu=\mathrm{R}\left(1+\alpha^{2}\right) /(4 \pi \mathrm{P})\right)$ for above threshold operation are also presented in the same figure. The average optical power $P$ used in this figure is numerically computed using the stationary parts of (1) and (4). The parameters used in the calculation to obtain Fig. 1 are: $\alpha=6, B=10^{-16} \mathrm{~m}^{3} \mathrm{~s}^{-1}, v=10^{-16} \mathrm{~m}^{3}, B_{\mathrm{sp}}=2.8 \times$ $10^{-5}, G_{N}=5.6 \times 10^{3} \mathrm{~s}^{-1}, N_{0}=10^{8}$, and $\alpha_{0}=6.372 \times$ $10^{11} \mathrm{~s}^{-1}$. In the region well below threshold, where the phase-amplitude coupling is negligible, the linewidth decreases with the injection current in accordings with the Schawlow-Townes formula [1], [2]. As the threshold is approached, the material gain is more and more restricted to its threshold value and the phase-amplitude coupling becomes more and more important. The linewidth changes gradually from what is predicted by the Schawlow-Townes formula to the modified Schawlow-Townes formula [2]. In Fig. 1, a local minimum of the linewidth is achieved at the current $I \approx 0.991 I_{\text {th }}$ and then a local maximum is reached with the injection current just above the operating threshold. This local minimum sets a limitation on the minimum optical bandwidth achievable in the resonant type semiconductor laser amplifier. This is in contrast to the conventional concept that the narrower small-signal optical bandwidth can be obtained by indefinitely approaching

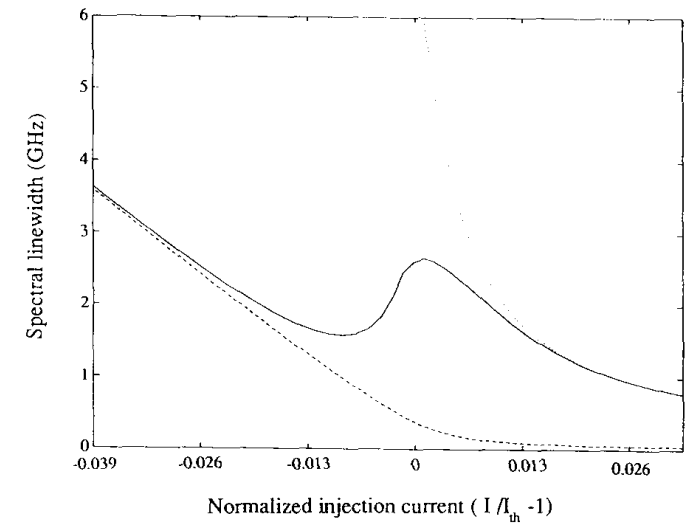

Fig. 1. Calculated spectral linewidth (solid line) with $\alpha=6$, and $B_{\mathrm{sp}}=$ $2.8 \times 10^{-5}$. The dashed line and the dotted line indicate the linewidths predicted by the Schawlow-Townes formula and the modified Schawlow-Townes formula, respectively.

the threshold point [6]-[8]. Increase in both $\alpha$ and the spontaneous emission factor $B_{\mathrm{sp}}$ will enlarge the transition region and increase the local minimum and maximum of the linewidth. The width of the transition region versus the linewidth enhancement factor $\alpha$ is shown in Fig. 2, where the width of the transition region is defined by the injection-current difference between the local minimum and the local maximum of the linewidth. The linewidth values at the local minimum and the local maximum versus $\alpha$ are reported in Fig. 3. Quasi-linear relationships have been found. When the value of $\alpha$ is less than 3 , the linewidth difference between the local minimum and the local maximum disappears gradually and the linewidth versus current curve tends to be monotonic. Since the local minimum of the linewidth is equivalent to the minimum small-signal optical bandwidth achievable in a semiconductor laser amplifier, this means that a semiconductor laser with low linewidth enhancement factor and low spontaneous emission factor is preferable in narrowband optical filter applications, e.g., a laser with quantum-well structure.

\section{EXPERIMENT}

In order to verify the theoretical prediction, the linewidth of a DFB-BH semiconductor laser has been measured using the standard heterodyne technique. Another DFB-BH laser biased at three times that of the threshold current was used as the local oscillator with the linewidth less than $20 \mathrm{MHz}$. Both lasers were temperature and current stabilized and each of them was optically isolated (by more than $70 \mathrm{~dB}$ ) with a Faraday optical isolator. A p-i-n photodiode and a microwave spectrum analyzer were used to monitor the heterodyne IF spectrum and to evaluate the laser linewidth. A monochromator is used to see the mode structure and to evaluate the linewidth of the laser operating well below threshold. The threshold current of the test laser was $21.9 \mathrm{~mA}$. The measured spectral linewidth versus the normalized injec- 


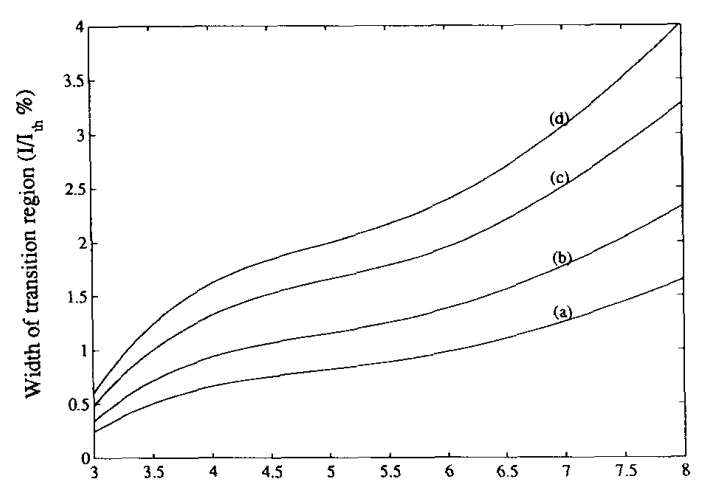

Linewidth enhancement factor $\alpha$

Fig. 2. Width of the transition region versus the linewidth enhancement factor $\alpha$ for different values of spontaneous emission factor $B_{\mathrm{sp}}=$ (a) $2.8 \times 10^{-5}$, (b) $5.6 \times 10^{-5}$, (c) $1.12 \times 10^{-4}$, and (d) $1.68 \times$ $10^{-4}$.

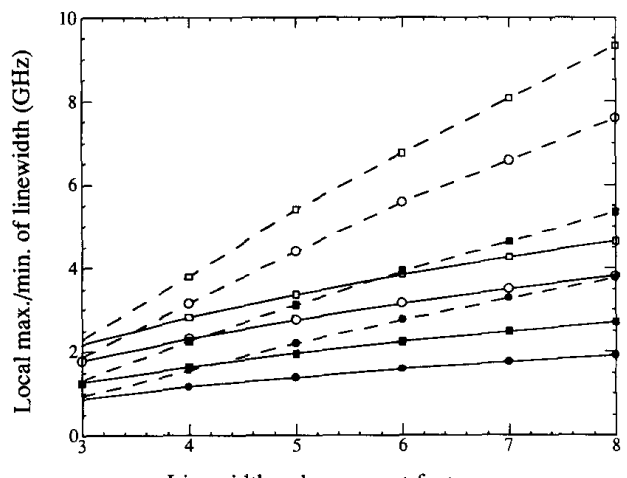

Linewidth enhancement factor $\alpha$

Fig. 3. Local minimum linewidth (solid lines) and local maximum linewidth (dashed lines) versus the linewidth enhancement $\alpha$ for different values of spontaneous emission factor $B_{\mathrm{sp}}=$ (black circles) $2.8 \times$ $10^{-5}$, (black rectangles) $5.6 \times 10^{-5}$, (white circles) $1.12 \times 10^{-4}$ and (white rectangles) $1.68 \times 10^{-4}$.

tion current is shown in Fig. 4. In comparing Fig. 4 with Fig. 1, qualitative agreement is evident. The minimum linewidth was obtained at $98.6 \%$ of the threshold current as shown in Fig. 4.

\section{CONCLUSION}

The linewidth of a single mode semiconductor laser operating in the threshold region has been studied both theoretically and experimentally. Due to the strong phase-amplitude coupling in semiconductor lasers, the linewidth decreasing with the injection current is not monotonic in the threshold region. The local minimum just below threshold sets a limitation in the minimum optical bandwidth achievable in the semiconductor laser narrow-band optical filter application. Lasers with low linewidth enhancement factor and low spontaneous emis-

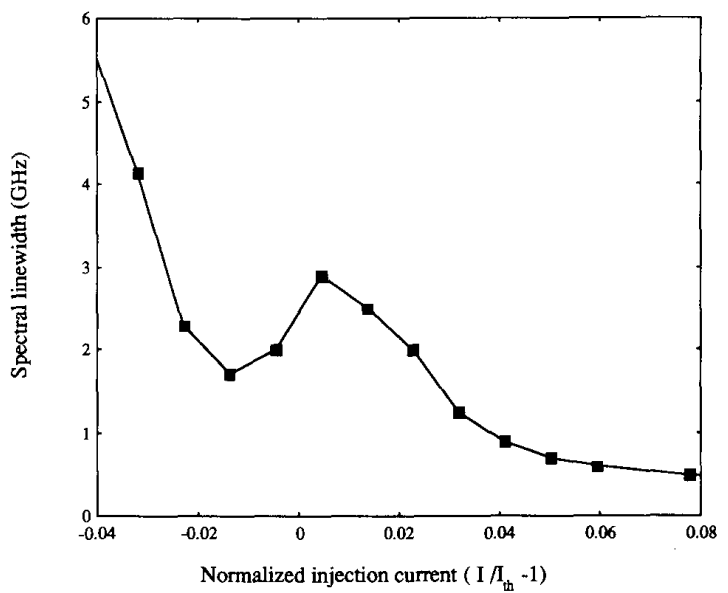

Fig. 4. Measured spectral linewidth versus the normalized injection current for a conventional single-mode DFB-BH semiconductor laser.

sion factor are found to be suitable for narrow-band optical filter applications.

\section{ACKNOWLEDGMENT}

The authors wish to thank P. Gambini, M. Puleo, and E. Vezzoni of the CSELT for the help in the experiment. A. P. Ansbro is also acknowledged for help in the computer program and for reading the manuscript.

\section{REFERENCES}

[1] A. L. Schawlow and C. H. Townes, "Infrared and optical lasers," Phys. Rev., vol. 112, pp. 1940-1949, 1958.

[2] C. H. Henry, "Phase noise in semiconductor lasers," J. Lightwave Technol., vol. LT-4, pp. 298-311, 1986.

[3] Z. Toffano, A. Destrez, C. Birocheau, and L. Hassine, "New linewidth enhancement determination method in semiconductor lasers based on spectrum analysis above and below threshold," Electron. Lett., vol. 28, pp. 9-11, 1992.

[4] M. Lax, "Classical noise V: Noise in self-sustained oscillators," Phys. Rev, vol. 160, pp. 290-307, 1967.

[5] R. D. Hempstead and M. Lax, "Classical noise VI: Noise in self-sustained oscillators near threshold," Phys. Rev., vol. 161, pp. 350-366, 1967.

[6] H. Risken and K. Seybold, "Linewidth of a detuned single mode laser near threshold," Phys. Lett., vol. 38A, pp. 63-64, 1972.

[7] K. Magari, H. Kawaguchi, K. Oe, and M. Fukuda, "Optical narrow-band filters using optical amplifications with distributed feedback," IEEE J. Quantum Electron., vol. 24, pp. 2178-2190, 1988.

[8] H. Nakajima, "High-speed and high-gain optical amplifying photodetection in a semiconductor laser amplifier," Appl. Phys. Lett., vol. 54, pp. 984-986, 1989

[9] F. S. Choa and T. L. Koch, "Static and dynamical characteristics of narrow-band tunable resonant amplifiers as active filters and receivers," J. Lightwave Tech., vol. LT-9, pp. 73-83, 1991

[10] L. G. Kazovsky, M. Stern, S. G. Menocal, Jr., and C. E. Zha, "DBR active optical filters: transfer function and noise characteristics," $J$. Lightwave Technol., vol. LT-8, 1331-1451, 1986.

[11] N. Tessler, R. N. Eisenstein, J. Salzman, U. Koren, G. Raybon, and C. A. Burrus, "Distributed Bragg reflector active optical filters," IEEE J. Quantum Electron., vol. 27, pp. 2016-2024, 1991.

[12] M. Lax, "Fluctuation and coherence phenomena in classical and quantum physics," Bradneis University Summer Institute in Theoretical Physics 1966, Vol. 2, M. Chretian, E. P. Gross and S. Desar, Eds. New York: Gordon and Breach, 1966, p. 293. 